\title{
Extinguishing Conditioned Responses During Opiate Dependence Treatment Turning Laboratory Findings into Clinical Procedures
}

\author{
A. Thomas Mclellan, PhD, Anna Rose Childress, PhD, \\ Ronald Ehrman, PhD, Charles P. O'Brien, MD, Phd
}

Philadelphia Veterans Administration Medical Center and the University of Pennsylvania School of Medicine

\author{
AND \\ SteVen Pashro, PhD \\ VA Medical Center, Coatesville, PA
}

\begin{abstract}
Former opiate addicts (even those who have remained drug-free for several months) often report symptoms of opiate withdrawal (eg. nousea, gooseflesh, etc.) and/or intense drug craving when exposed to stimuli previously associated with the act of drug injection. This phenomenon of learned or "conditioned" withdrawal/craving is widely reported and is potentially important in explaining relapse to drug use. However, no effective, clinically applicable intervention had been available to "extinguish" these conditioned phenomena. An ongoing project to develop such an intervention has nevealed:

1. Conditioned withdrawal and craving are pervasive among both methadone maintained patients (even though actual physical withdrawal is blocked) and drug-free patients even after 30 days of inpatient Therapeutic Community rehabilitation.

2. Conditioned withdrawal and craving can be effectively extinguished in an intensive, threeweek, inpatient procedure.

3. Emotional states such as anger, depression and anxiety can elicit and exacerbate conditioned withdrawal and craving. They may also act as an integral part of a conditioned stimulus complex.

The authors discuss the problems associated with turning a laboratory-based procedure into a clinical intervention. Encouraging preliminary results from an integrated treatment "package" are presented.
\end{abstract}

Key Words-Addiction, extinction, desensitization, conditioned responses, craving

\section{INTRODUCTION}

OVER THE PAST 10 years, our research group has investigated a wide range of variables associated with the etiology and treatment of opiate dependence. While many individual factors have been explored, our recent investigations have focused on two major areas that appear to be importantly involved in the

This work was supported by VA Project \$704 and by NIDA Project \#DA03008.

Requests for reprints should be sent to Dr. McLellan, Bld. \#7, Phila. VAMC, Phila., PA 19104. initial development of habitual use and especially in relapse following treatment. In one series of investigations we explored the role of psychiatric factors in opiate dependence. We and others in the field (Woody, O'Brien, \& Rickels, 1975; Dorus \& Senay, 1980; Rounsaville et al., 1982) were impressed by the prevalence of depression and anxiety disorders in our opiate-abusing population and the role these factors played in promoting relapse to drug use. These observations led directly to a 4 year evaluation of adjunctive psychotherapy within a standard methadone maintenance program (Woody et al., 1983). Results during the course of treatment and at both the seven 
and twelve month follow-up points, indicated significantly better overall outcomes in the therapy patients than in those who received standard counseling alone (Woody et al., 1983).

This psychotherapy investigation was a natural outgrowth of our clinical observations of psychiatric symptoms in opiate abusers and the ready availability of standard forms of psychotherapy that had previously been shown to be effective with nondrugdependent patients. Thus, in this case the task was to adapt a standard treatment (psychotherapy) to this population and then evaluate it systematically against another standard treatment that is commonly used with opiate addicts (counseling).

Our second area of investigation has focused on the role of learned or "conditioned" factors in opiate dependence. These investigations also originated from clinical observations of detoxified patients who had returned to the area where they had previously obtained and used drugs. These patients sometimes reported symptoms of opiate withdrawal (eg. nausea, goose flesh, muscle aches, etc.) and drug craving, apparently elicited by the sights, sounds and odors associated with prior drug use. These phenomena occurred despite the fact that the patients had been drug-free for many months.

Wikler explained these withdrawal phenomena as the result of a classically conditioned association between the actual pharmacological withdrawal often experienced by street addicts and a large constellation of drug-related stimuli (eg. drug talk, drug buying, syringes, etc.) repeatedly paired with this state of withdrawal (Wikler, 1973). More recent work has shown that even stimuli paired explicitly with drug administration and not withdrawal, can also elicit physiological signs of withdrawal; and these have been considered conditioned "compensatory responses" (Siegel, 1976). Whatever their origins, these "conditioned withdrawal responses" can mimic the typology and severity of actual opiate withdrawal. These conditioned withdrawal responses are more frequent and apparently more persistent than the conditioned euphoric or "high" responses that have also been reported (Ternes et al., 1980), and thus could be important factors in explaining relapse to drug use.

We therefore began a series of laboratory studies designed to investigate the parameters of these conditioned responses in humans. In these laboratory studies, we discovered that naloxone-precipitated withdrawal could be used to produce a learned or conditioned opiate withdrawal response to stimuli that had been paired with it; and that this conditioned withdrawal response showed many of the physiological signs which occur in regular opiate withdrawal (O'Brien, Testa, O'Brien, \& Brady, 1977). Finally, we found that opiate addicts, but not normal volunteers, showed physiological signs of withdrawal following presentation of drug-related videos, slides and objects (Ternes et al., 1980).

These laboratory-based experiments showed the reproducibility of these phenomena and provided additional suggestions that the conditioned factors could be important in relapse. However, unlike our investigations of psychiatric factors in opiate dependence, there were no "ready-made" treatments for symptoms of conditioned withdrawal and craving. Further, we quickly found out that the transition from laboratory observation to clinical procedure is complicated and often impractical.

\section{"Extinction Trials as a Laboratory Procedure"}

In our first clinical attempt to address the conditioned factors in opiate dependence, we approached the problem using extinction procedures directly from the laboratory. Our reasoning was simple and direct: If the sights and smells associated with cooking up and injecting opiates were able to elicit opiate craving and withdrawal through their history of paired association with drug use, we would simply break this association by having these same stimuli and procedures presented but not followed by the opiate. These presentations would therefore be extinction trials. To this end, we paid drug-free subjects to repeatedly cook-up and inject saline, or opiate (in subjects protected by naltrexone) (Ternes, O'Brien, Greenstein, \& McLellan, 1980B) to test the "extinction" procedure as a clinical intervention.

The "cook-up" and self-injection procedure was at first reported to be pleasant but after only a few trials, the self-injections produced withdrawal reactions. The subjects uniformly disliked this situation, with the majority of them refusing to continue past six to ten extinction trials. Although the follow-up results from this small group suggested somewhat better outcomes in those patients who had received the "extinction" trials, it was clear that the procedure was so aversive that no patient would participate long enough to permit a complete extinction of the conditioned withdrawal responses. The results of this initial effort suggested that we had been naive in our direction translation of a laboratory phenomenon into a clinical procedure.

\section{"Extinction" Trials as a Clinical Procedure}

Realizing that the act of cooking up and injecting an inert substance was too aversive for most patients, we decided to modify the original procedure by developing a graduated hierarchy of conditioned stimulus presentations that would permit the subject to build up tolerance for the cook-up procedure (injection was made optional) and would also permit non-reinforced presentation of other antecedant drug-related stimuli. 
As an additional means of fostering acceptance of these evocative stimuli, we decided to follow each presentation with a period of deep relaxation training, under the view that the simple presentation of these stimuli without any other intervention might trigger intense craving and actually increase the likelihood of drug use after the session.

This second version of our extinction procedure resembled the clinical practice of "systematic desensitization" (Wolpe, 1958). This clinical-behavioral procedure has been effective in reducing phobic responses by initially exposing the patient to stimuli that are less evocative and thus better tolerated than the target stimulus. Further, deep relaxation exercises and/or individual therapy are generally used to reduce the anxiety, tension and other uncomfortable emotional responses produced by exposure to these stimuli.

Given what we thought was a more practical and potentially effective procedure for addressing these conditioned responses, we decided to evaluate the procedure as part of a multi-group study incorporating psychotherapy, extinction and relaxation training. The design of this second version of our integrated treatment study has been detailed in other papers (Childress, McLellan, \& O'Brien, 1984; 1985). Here we will describe the study design briefly and present some clinical outcome data from this stage of the procedure.

Subjects. In this stage of our efforts we decided to implement the procedure in our outpatient methadone maintenance population. This had been the population used in our earlier, successful psychotherapy study. We also expected that we would be able to measure the effects of the extinction trials by lowered methadone dose, reduced use of illicit drugs and success at achieving abstinence. Patient volunteers were recruited through direct contact or referral from their drug counselor. All patients were clinically screened to rule out DSM III diagnoses of psychosis or organicity.

Eligible patients from this program were randomly assigned to one of three treatment groups. Patients in the experimental group received cognitive-behavioral psychotherapy plus graduated extinction trials and relaxation (CE group). One control group received cognitive-behavioral therapy and relaxation but no extinction (CT group), while a second control group received standard drug counseling and educational/ control materials (DC group). Professional attention, session length, and small payments contingent upon session attendance were equivalent for all treatment groups.

From the 173 subjects who were formally contacted, 88 patients $(51 \%)$ actually agreed to participate. Refusal to participate was generally due to an inability to arrange for time from work or because they felt the time commitments were too demanding. Twenty-two $(25 \%)$ of the 88 patients who initially agreed to participate never followed through with initial screening procedures. Ten $(13 \%)$ of the 88 patients who agreed to participate were screened out of the study due to an organic or psychotic disorder.

The remaining 56 patients were randomly assigned to the three treatments. We arbitrarily considered any patient who completed less than ten sessions $(n=21)$ as a drop-out. The drop-out rate was approximately equal across the three treatment groups with $7(33 \%)$, $6(29 \%)$ and $8(38 \%)$ patients dropping out of the $\mathrm{CE}, \mathrm{CT}$ and $\mathrm{DC}$ groups respectively.

Procedure Each hour-long session for patients in the extinction (CE) group began with $\mathbf{3 0}$ minutes of psychotherapy, followed by approximately 10-15 minutes of exposure to extinction stimuli. Each session ended with 15-20 minutes of relaxation training, guided by audio cassette. The graduated hierarchy of extinction stimuli included self-produced verbal imagery ("drug stories"), audiotapes of drug talk, color slides of cook-up-injection rituals, videotapes of drug purchase, cook-up and injection, and finally, handling of drug objects in a cook-up/tie-off procedure. Saline self-injection, the final member of the extinction series, was encouraged but optional and no subject actually chose to inject. For each patient, the ordering of extinction stimuli across sessions was the same and a fixed trials procedure was used, which determined the number of exposures to each of the drug-related stimuli. Extinction sessions were conducted three times weekly, with 35 sessions comprising a complete course of treatment. Daily methadone was administered immediately after sessions so that its onset effects would not interfere with physiological or subjective measures.

\section{MEASURES}

\section{Psychological Tests}

The Beck Depression Inventory, the Maudsley Personality Inventory and the Hopkins Symptom Checklist, 90 were administered to each patient at the start of treatment and at the one and six-month follow-up points.

\section{Structured Interviews}

The Addiction Severity Index (ASI) interview was administered to all patients at the start of treatment and again at the one and six-month evaluation points. The ASI is a clinical/research interview, designed to assess problem severity in seven areas of functioning commonly impaired in drug-dependent patients: medical, employment/support, drug abuse, alcohol abuse, 
legal, family/social, and psychological (McLellan, Luborsky, Woody, \& O'Brien, 1980). In each of these areas, both objective and subjective questions are asked to measure the number, extent, and duration of problem symptoms in the patient's lifetime and in the past 30 days. Sets of related items from each of the problem areas are standardized and summed to produce factor scores that provide reliable and valid general estimates of problem severity at each evaluation point. It is important to note that the ASI interviews were done by independent technicians who were not part of the treatment staff.

\section{RESULTS}

\section{Within-Group Improvement Comparisons}

As an initial assessment of the improvement shown by each group, we compared their pre-treatment and one month post-treatment scores on a range of ASI measures, using the paired t-test procedure. All measures were based upon the 30 day periods preceeding the start of treatment and the 6 month evaluation. Higher factor scores indicate greater problem severity. As can be seen from the Table 1 data there was general improvement shown by most groups in most measures.

The CE group showed improvements in 18 of 21 measures with six reaching statistical significance ( $p=.05$ or less) and one showing a trend toward significance $(p=.08)$. A highly significant $(p<.01)$ improvement was shown in the alcohol use factor score, while other significant $(p<.05)$ changes were seen in the areas of drug use, psychiatric status and family relations. However, only minor improvements were seen in employment and legal status for this group.

The CT group showed improvements in 20 of the 21 measures, with seven of these reaching statistical significance $(p=.05)$ and one showing a trend $(p=.07)$. Major changes $(p<.01)$ were seen in the drug, alcohol and family factor scores. Additional changes were seen in psychiatric status although there was very little improvement shown in legal status or employment.

The DC group improved in 9 of the 21 measures with two reaching significance $(p<.05)$ and one showing a trend $(p<.07)$. This group showed significant changes in employment status and some improvement in legal status and drug use. However, there was no change in the areas of alcohol use, psychiatric status or family relations.

\section{Between-Group Outcome Comparisons}

As an additional comparison of the three groups we performed an analysis of covariance (ANCOVA) on

TABLE 1

Patient Improvement

\begin{tabular}{|c|c|c|c|c|c|c|c|c|}
\hline & \multicolumn{2}{|c|}{$\begin{array}{c}C E \\
(N=13)\end{array}$} & \multicolumn{3}{|c|}{$\begin{array}{c}\text { CT } \\
(N=11)\end{array}$} & \multicolumn{2}{|c|}{$\begin{array}{c}D C \\
(N=11)\end{array}$} & \multirow{2}{*}{$\begin{array}{l}\text { ANCOVA ON 6-MO } \\
\text { SCORES } \\
p=\text { Group Comp. }\end{array}$} \\
\hline & Adm. & 6-Mo. & Adm. & & 6-Mo. & Adm. & 6-Mo. & \\
\hline $\begin{array}{l}\text { Medical factor } \\
\text { Days of med. prob. }\end{array}$ & $\begin{array}{r}496 \\
2\end{array}$ & $\begin{array}{r}370 \\
1\end{array}$ & $\begin{array}{r}453 \\
2\end{array}$ & & $\begin{array}{r}-243 \\
0\end{array}$ & $\begin{array}{r}519 \\
2\end{array}$ & $\begin{array}{r}624 \\
3\end{array}$ & $\begin{array}{l}.02 \mathrm{CT}, \mathrm{CE}<\mathrm{DC} \\
.03 \mathrm{CT}, \mathrm{CE}<\mathrm{DC}\end{array}$ \\
\hline $\begin{array}{l}\text { Employment factor } \\
\text { Days working } \\
\text { Money earned }\end{array}$ & $\begin{array}{r}383 \\
3 \\
97\end{array}$ & $\begin{array}{r}354 \\
5 \\
154\end{array}$ & $\begin{array}{r}345 \\
5 \\
266\end{array}$ & & $\begin{array}{r}280 \\
8 \\
+372\end{array}$ & $\begin{array}{r}370 \\
4 \\
160\end{array}$ & $\begin{array}{r}* 298 \\
7 \\
* 327\end{array}$ & $\bar{z}$ \\
\hline $\begin{array}{l}\text { Drug use factor } \\
\text { Days opiates } \\
\text { Day stimulants } \\
\text { Days depressants }\end{array}$ & $\begin{array}{r}243 \\
3 \\
1 \\
19\end{array}$ & $\begin{array}{r}194 \\
6 \\
1 \\
* 9\end{array}$ & $\begin{array}{r}184 \\
4 \\
2 \\
7\end{array}$ & .07 & $\begin{array}{r}+133 \\
4 \\
0 \\
2\end{array}$ & $\begin{array}{r}223 \\
3 \\
1 \\
3\end{array}$ & $\begin{array}{r}221 \\
4 \\
0 \\
2\end{array}$ & $\begin{array}{l}.02 \mathrm{CT}, \overline{\mathrm{DC}}<\mathrm{CE} \\
.04 \mathrm{CT}<\mathrm{DC}, \mathrm{CE}\end{array}$ \\
\hline $\begin{array}{l}\text { Alcohol use factor } \\
\text { Days drinklng } \\
\text { Days intoxicated }\end{array}$ & $\begin{array}{r}184 \\
12 \\
7\end{array}$ & $\begin{array}{r}+126 \\
* 6 \\
4\end{array}$ & $\begin{array}{r}163 \\
8 \\
5\end{array}$ & & $\begin{array}{r}160 \\
7 \\
4\end{array}$ & $\begin{array}{r}117 \\
8 \\
4\end{array}$ & $\begin{array}{r}123 \\
10 \\
2\end{array}$ & $.06 \mathrm{CE}, \overline{\mathrm{CT}}<\mathrm{DC}$ \\
\hline $\begin{array}{l}\text { Legal factor } \\
\text { Crime days } \\
\text { lllegal income }\end{array}$ & $\begin{array}{r}60 \\
3 \\
81\end{array}$ & $\begin{array}{r}40 \\
1 \\
25\end{array}$ & $\begin{array}{r}65 \\
4 \\
120\end{array}$ & & $\begin{array}{r}90 \\
7 \\
140\end{array}$ & $\begin{array}{r}68 \\
3 \\
103\end{array}$ & $\begin{array}{r}0 \\
0 \\
0\end{array}$ & $\begin{array}{l}.02 \mathrm{DC}, \overline{\mathrm{CE}}<\mathrm{CT} \\
.04 \mathrm{DC}, \mathrm{CE}<\mathrm{CT}\end{array}$ \\
\hline $\begin{array}{l}\text { Psychiatric factor } \\
\text { Days psych. prob. } \\
\text { Beck depression inv }\end{array}$ & $\begin{array}{r}258 \\
7 \\
11\end{array}$ & $\begin{array}{r}-155 \\
5 \\
8\end{array}$ & $\begin{array}{r}206 \\
5 \\
12\end{array}$ & & $\begin{array}{r}* 92 \\
2 \\
* 7\end{array}$ & $\begin{array}{r}209 \\
7 \\
10\end{array}$ & $\begin{array}{r}298 \\
10 \\
10\end{array}$ & $\begin{array}{l}.04 \mathrm{CT}, \mathrm{CE}<\mathrm{DC} \\
.04 \mathrm{CT}<\mathrm{CE}<\mathrm{DC} \\
.07 \mathrm{CT}<\mathrm{CE}, \mathrm{DC}\end{array}$ \\
\hline
\end{tabular}

1. All criteria were measured during the 30 days before study start and six months after the last treatment session. Higher factor scores indicate greater problem severity.

$t=p<.05^{*}=p<.01$ by paired $t$-test.

2. Covariate was the admission score. The results of paired group comparisons are shown with the better outcomes at the left. 
the one-month follow-up scores, using the pre-treatment measure as the covariate. This analysis permits the comparison of group outcomes adjusting for initial differences in their pre-treatment values. Results of this analysis can be seen in the last column of Table 1. A significant between-groups main effect at the $p<.05$ level was followed by paired group comparisons among the adjusted means.

Significant $(p \leq .05)$ main effects were seen on 8 of the 21 analyses, particularly in the areas of alcohol and psychiatric status, crime and psychiatric status. The DC group had the poorest adjusted outcomes on all of the eight comparisons $(p<.05)$. The two experimental groups (CE, CT) did not differ from each other on any measure $(p>.10)$.

As a final analysis of between-group performance, we ranked the adjusted outcome scores on all 21 measures and performed a chi square test on the rankings. The results of this general comparison showed a significant overall effect (chi square $=12.34 d f=4$, $p<.02)$, with the DC group significantly $(p<.03)$ worse than the other two groups, which did not differ from each other $(p>.10)$.

\section{DISCUSSION}

The most apparent result from this early work was that although both the psychotherapy plus extinction (CE) and psychotherapy alone (CT) groups had generally better 6 month outcomes than the drug counseling group (DC), there was no evidence that the addition of the extinction procedure (at least as we had performed it) had made a significant contribution beyond the psychotherapy. Careful examination of the data suggested a need for further modification in our conceptual approach and our procedures. These are detailed below.

\section{Why Did We Attempt Extinction in a Methadone Maintenance Sample?}

As discussed earlier, we had initially conceived of the idea of integrating the laboratory-based extinction procedures with an existing program of psychotherapy. We, perhaps naively, expected that the extinction procedure could be effectively applied on an outpatient basis and that if effective, it would have pervasive, measurable results on several aspects of patients' performance such as increased employment, lowered methadone dose, negative urinalysis results. In fact, our earlier studies had shown that psychotherapy could produce these general improvements (Woody et al., 1983) and we had hoped that the addition of the extinction module could produce cumulative benefits.

In reconsideration, it is now clear that, unlike psychotherapy, which has a broad focus and a wide spectrum of direct effects, the extinction procedure is narrowly focused on those stimuli in the environment which may produce conditioned drug craving and withdrawal symptoms. Further, the only direct effect of this procedure that can reasonably be expected is the continuation and extension of drug-free status in a drug-free patient.

\section{How Effective Was the Extinction Component?}

As described earlier, our original observations and laboratory findings focused on the physiological components of a conditioned withdrawal phenomenon, i.e. the lowered skin temperature, the shallow, rapid respiration, the increased heart rate, and the increased arousal. For this reason we wanted to expose the patients to stimuli that we knew would produce these physiological changes: the cook-up/injection procedure. The stimulus hierarchy was designed to gradually enable the subject to tolerate exposure to the most evocative cues.

In fact, our initial data suggest that some subjects did show reductions in their conditioned physiological responses to the drug related stimuli presented in the laboratory test situation; while other subjects showed evidence of reduced subjective reports of craving and/ or withdrawal responses, over the course of the extinction sessions (see Childress, McLellan, \& O'Brien, 1984, 1985). However, it must be stated that these reductions were not profound, nor did they occur in a majority of subjects. Thus, it appears that the extinction intervention, as delivered in this experiment, was not effective.

\section{What Did We Learn From Our Efforts?}

During the course of actually performing these extinction trials, three facts became apparent that suggested a basis for improvement in our next attempt at extinction. First, several subjects showed greater responsiveness to some of the audio and video stimuli related to drug procurement than to the cook-up/injection procedure. While order effects cannot be ruled out, this finding suggests that the most evocative stimuli may often be idiosyncratic to the individual and to his particular addiction history. For many individuals, the receipt of a paycheck or even the sight of an anti-drug-use poster may be associated with more profound conditioned responses than the standardized stimuli used in our project. We now realize that in order to be maximally effective, an extinction procedure must identify and focus on the particular sights, sounds, odors, and situations that appear to be individually arousing to each subject.

We had originally hypothesized that conditioned changes in physiological signs of withdrawal might be the basis for subjects' reports of subjective with- 
drawal symptoms, and that in turn, these subjective symptoms of withdrawal would be the basis for the additional subjective response of drug craving. However, the second finding from our project was that subjective reports of craving and withdrawal symptoms did not correlate well with concurrently recorded changes in the physiological measures of heart rate, respiration, GSR and skin temperature (multiple $R=.23$ ). For example, it was common to have a marked physiological response to a stimulus with no report of subjective craving or withdrawal. Similarly, it was often the case that craving was reported without measurable physiological changes or even subjective reports of withdrawal. In addition, our data indicate that even the three subjective measures recorded (high, withdrawal, craving reports) were poorly intercorrelated (Ave. $R=.19$ ) across and within subjects. Thus, patients would regularly report that drug-related stimuli would elicit subjective feelings of craving without concommittant subjective reports of withdrawal or high-like symptoms.

Of course, we recognize that the peripheral measures of physiological change used in this study may not detect significant central physiological effects which may be well-correlated with subjective craving. Further, it should be noted that results of this nature are commonly seen in studies investigating the concurrence of physiological and subjective effects in depression, hunger, thirst and other emotional and motivational states (Schachter, 1968; Cannon, 1975). However, the lack of a strong relationship between the supposed physiological bases of the subjective response of drug craving, as well as the weak relationship among the three subjective measures suggests that the multiple conditioned responses seen in our patients (objective physiological changes and subjective reports of high, withdrawal and craving) may not be part of a single phenomenon and therefore should be addressed individually in an expanded extinction procedure.

The third finding resulting from our extinction procedure was that cognitive and emotional factors may have a powerful effect on the expression of any conditioned response. The effects of cognitive set were seen in one subject, who showed significant physiological changes during his initial exposure to the cook-up procedure. However, on the second day of exposure, he exhibited essentially no response to these same cues. When the subject was questioned regarding the situation, he responded that he controlled his response to the cues by concentrating on the fact that "the situation wasn't real." This ability to change the profound effects of stimuli on both conditioned physiological and subjective responses illustrates the importance of cognitive set in eliciting conditioned responses.

A second incident provides an even more graphic example of how emotional factors may interact with conditioned stimuli to alter responsiveness. In this incident, a patient had undergone the complete stimulus hierarchy procedure and had reported a gradual disappearance (extinction) of drug craving and subjective withdrawal responses. He had experienced seven trials where the cook-up procedure produced no measurable subjective or physiological change. Prior to the last session, the patient was involved in a lengthy argument with a hospital security guard. The patient was still angry as he entered the extinction session. Under this emotional condition the patient experienced a re-emergence of severe craving and withdrawal when he performed the cook-up procedure, surprising even himself. Anecdotally, patients often report that they can walk by an area where they have previously bought or used drugs without experiencing feelings of craving or withdrawal. However, when angered, depressed or anxious, these same stimuli can trigger intense craving and/or withdrawal-like symptoms.

There are several possible interpretations of this interaction. We have chosen to view it as an example of a compound CS. That is, drug-related stimuli in the presence of a particular emotional state may constitute a compound conditioned stimulus, capable of eliciting a maximal conditioned response (Pavlov, 1960). We have long been impressed by the impact of emotional states and emotional problems on relapse to drug use. Patients often use drugs to alleviate negative affective states and our original psychotherapy intervention study was based on these observations. This additional data suggests that the emotional state of the individual may also trigger drug use in another way; by acting as a conditioned interoceptive stimulus which in conjunction with conditioned exteroceptive stimuli in the environment, elicit conditioned physiological signs of withdrawal and conditioned subjective symptoms of drug craving.

In summary, we found that the extinction procedure was inappropriate in our methadone-maintained sample, due to several practical constraints and we conclude that the procedure is best suited to a drugfree population. Second, we found that the physiological and subjective conditioned responses elicited by drug-related stimuli are not well correlated and should be addressed independently. Third, we saw the powerful effects of cognitive and emotional factors on conditioned responses.

\section{A Revised Design For A More Effective Extinction Procedure}

Based on these three findings we have developed a revised procedure which shows preliminary evidence of being more effective in reducing the full range of conditioned responses produced by drug-related stimuli. In this third stage of the ongoing project we have 
directed our efforts exclusively toward abstinent patients. These patients are referred directly from a 30day inpatient, drug-free therapeutic community program. The three original groups have been retained as well as a fourth which receives standard counseling plus extinction. The revised procedure is conducted in two phases. During the 3 week inpatient phase, extinction subjects hear an audiotape and view a videotape involving drug related activities, then cook-up and prepare to (but not actually) inject an opiate. This series of stimuli is repeated three times in each of 20, hour-long, extinction sessions during the 3 week period, for a total of 60 stimulus exposures. This inpatient phase permits intensive "massed-trials" extinction without the danger of re-exposure that would occur in an outpatient setting. In addition, the extinction subjects undergo the hypnotic mood induction of four mood states (anger, depression, anxiety and euphoria) during which they perform the cook-up procedure. This enables identification of those moods which combine with the exteroceptive stimuli to produce the most intense conditioned responses. All subjects also receive twelve sessions of either psychotherapy or drug counseling during the 3 week inpatient phase.

Following the inpatient phase of the project, subjects are referred to outpatient treatment where they meet with their counselor or therapist weekly for 8 weeks. Extinction subjects begin exposure to the particular idiosyncratic stimuli from their prior history which elicit conditioned responses. These stimuli are presented repeatedly while the subject is hypnotically induced into his most evocative moods (as determined during the inpatient phase).

\section{Preliminary Results from the Revised Extinction Procedure}

We have now completed the inpatient phase of our revised extinction procedure with eight drug-free patients referred directly from our inpatient therapeutic community. Most notable in this small group is the intense level of conditioned responding seen on initial exposure to the drug-related stimuli. Our impression is that these responses are more pervasive and intense than in our methadone maintained patients, despite the amount of treatment and the duration of drugfree status in these patients. Unsolicited comments from several of these patients following highly evocative initial sessions. were that ". . . it was as if I had never gone (to the T.C.). . . " These comments reinforce our view that these conditioned responses may be important but overlooked contributors to drug relapse.

Results from the full course of inpatient extinction already suggest the revised extinction procedure is more powerful and effective than the earlier attempts. Despite the generally enhanced level of conditioned responding seen in these subjects, all have shown gradual reductions in both physiological signs of conditioned withdrawal and in the subjective reports of conditioned withdrawal symptoms and drug craving. However, it should be noted that these response reductions occured only after 8 to 10 days of intensive extinction sessions (approximately 45-60 stimulus exposures). Post-treatment follow-up sessions will determine the extent to which these conditioned responses show spontaneous recovery.

Preliminary data from the hypnotic mood-induction component of the procedure are equally promising. The majority of subjects have been successfully induced into all four "test" moods, and several staff members have mastered the hypnotic procedures. As in our initial anecdotal reports, most patients have shown intensified levels of conditioned responding in one or two "problem" moods (usually anger and depression). Results of mood-induced extinction with individualized stimuli are not yet available but an expanded report of this interesting phenomenon is in preparation.

\section{CONCLUSION}

The latest attempt at integrating extinction with standard clinical treatment incorporates much of what we have learned in this continuing experimental process. We have targeted a more appropriate, drug-free subject population. The therapy and extinction components have been separated and individually designed. The extinction component now incorporates individualized stimuli more antecedent to the actual injection as well as intensified concentration on the cook-up procedure. Recognizing the importance of emotional state, we have incorporated a mood induction process as part of the extinction procedure which we hope will provide a more comprehensive method of addressing these conditioned factors.

We have learned much from this continuing process. In particular, we have learned that the transiation of a laboratory based discovery into a practical, effective clinical intervention is a complex process. Further, we have learned that the effects of conditioned phenomena in opiate dependent individuals are more diverse, individualized and pervasive than we initially anticipated.

\section{REFERENCES}

Cannon W.B. (1975). Bodily changes in pain, hunger, fear and rage, New York, Appleton Pub.

Childress A.R., McLellan A.T., \& O'Brien C.P. (1984). Meesurement and extinction of conditioned withdrawal-like responses in opiate dependent patients, Problems of Drug Dependence 1983, NIDA Research Monograph, USCPO.

Childress A.R., McLellan A.T., \& O'Brien C.P. (1985). Behavioral therapies for substance abuse. Int. J. Addict., 20, 947-969. 
Dorus W., \& Senay E. (1980). Depression, demographic factors and drug abuse, Am. J. Psychiat., 137, 24-36.

MeLellan A.T., Luborsky L., Woody G.E., \& O'Brien C.P. (1980). An improved evaluation instrument for substance abuse patients: The Addiction Severity Index, J. Nerv. Ment. Dis., 168, 26-33.

O'Brien C.P., Testa T., O'Brien T.J., \& Brady J.P. (1977). Conditioned narcotic withdrawal in humans, Science, 195, 1000-1002.

Pavlov I.P. (1960). Conditioned Reflexes, New York, Dover Pub., p. 141-148.

Rounsaville B.J., Weissman, M.M., Wilber C.H., \& Kleber, H. (1982). The heterogeneity of psychiatric diagnoses in treated opiate addicts, Anch. Gen. Psychiat., 39, 161-166.

Schachter S. (1968). Obesity and eating, Science 161, 751-756.

Siegel, S. (1976). Morphine analgesic tolerance: Its situation specificity supports a Pavlovian conditioning model, Science, 198, 323-325.
Ternes J.W., O'Brien C.P., and Grabowski J. (1980). Conditioned drug responses to naturalistic stimuli, Problems of Drug Dependence 1979, NIDA Research Monograph, USGPO.

Ternes J.W., O'Brien C.P., Greenstein R., McLellan A.T. (1980B). Unreinforced seif-injections: Effects on rituals and outcome in heroin addicts, Problems of Drug Dependence 1979, NIDA Research Monograph, USGPO.

Wikler A. (1973). Dynamics of Drug Dependence, Arch. Gen. Psychiat. 28, 611-616.

Wolpe J. (1958). Psychotherapy by Reciprocal Inhibition, Stanford, Calif. Stanford Univ. Press.

Woody G.E., O'Brien C.P. and Rickels K. (1975). Depression and anxiety in heroin addiets, Am. J. Psychiat., 32, 411-414.

Woody G.E., Luborsky L., McLellan A.T., and O'Brien C.P. (1983). Psychotherapy for opiate addicts: Does it help?, Arch. Gen. Psychiat. 40, 639-645. 